\title{
SPECTROSCOPIC MeASUREMENT OF TeXTILE FibReS
}

\author{
Hana Vaskova \& Jan Hrano
}
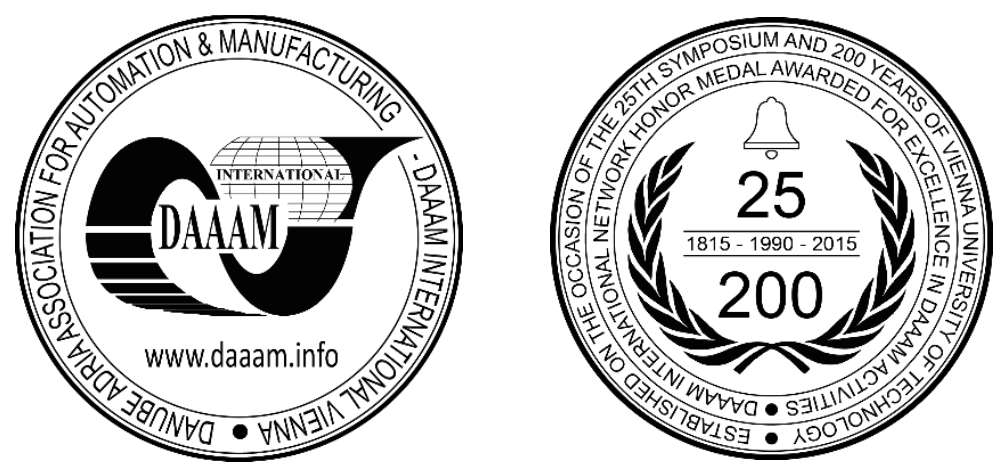

This Publication has to be referred as: Vaskova, H[ana] \& Hrano, J[an] (2017). Spectroscopic Measurement of Textile Fibres, Proceedings of the 28th DAAAM International Symposium, pp.0331-0333, B. Katalinic (Ed.), Published by DAAAM International, ISBN 978-3-902734-11-2, ISSN 1726-9679, Vienna, Austria DOI: $10.2507 / 28$ th.daaam.proceedings.045

\begin{abstract}
Raman spectroscopy as an effective tool for material identification offers benefits that are important for the process control of the fibres in terms of quality control in production, in use, but also in the field of fibre analysis for forensic identification. In particular, it is specific information on chemical composition and structure of material; rapidity of measurement, non-destructiveness, independence on chemicals, portability, possibility of automation of measurements and ability to detect structural changes. In the study, samples of natural and synthetic polymer fibres were measured and analysed. For the evaluation of large spectral data sets multivariate analysis techniques were applied to find the patterns within the data. The spectral database was created on the basis of the acquired Raman spectra of textile fibres and was successfully tested on other samples. The results show a great potential of the used method for the fibre identification.
\end{abstract}

Keywords: Quality; control; textile fibre; Raman spectroscopy; multivariate analysis techniques; spectral database

\section{Introduction}

People have been using textile fibres of a different nature since the beginning of their existence. Plant sources were used for the production of textile fibres more than twenty thousand years ago [1]. Textile production is an old craft that has been greatly changed by technological advances. Many types of fabrics come from new technologies and raw materials. Fabrics used today are very often a mixture of multiple materials. The palette of modern use emphasizes the properties of fibres and fabrics, such as durability, strength, breathability, flexibility, bacteria resistance, etc. [2]. Many tests/methods, from simple to sophisticate, are used to study, measure, verify and improve [3] modern fibres.

One of the main motivations for this research was to find and study method offering rapid, non-destructive measurement with the ability to recognize and identify unknown samples. Although microscopy is often the most powerful method [4], Raman spectroscopic examination gives valuable information on the structure of material. Lately, this method finds more and more applications across scientific fields. Its potential allows studying natural fibres based on cellulose [5], carbon-based structures [6], as well as biological systems, artworks or food. Raman spectroscopy offers benefits that are important for the process control in terms of quality control in production, in use [7], but also for forensic identification [4]. Because of its many advantages, Raman spectroscopy was chosen for the study of textile fibres and creation spectral database. Another motivation is related with forensic application. Fibres are considered as a one form of trace evidence [8]. They are transferred between the perpetrator and the victim or left behind at the crime scene during committing a crime. A functional spectral library is then a useful and effective tool for the identification. 


\section{Materials, Methods and Instrumentation}

The ability to obtain the relevant information on textile fibres is studied by a combination of Raman spectroscopic measurement with cluster analysis and principal component analysis.

\subsection{Textile fibres}

For the spectroscopic study of textile fibres, commonly used textile materials were chosen to cover various types of textiles. Twenty samples of natural and synthetic fibres, such as cotton, ramie, flax, wool, merino wool, silk, cellulose acetate, lyocell (rayon), viscose, nylon, polyamide and polyester were measured. A part of the mentioned materials included more samples. Therefore, duplicate samples were used to test the database. Colour of all used samples was natural/white.

\subsection{Raman spectroscopy}

Raman spectroscopy is a vibrational spectroscopic method providing specific chemical information of every single chemical substance and its modifications in the form of Raman spectra. A slight wavelength shifts occurring after irradiation facilitate the material identification and structural assessments. Substantial benefits arise from many advantages; method is rapid, non-destructive, contactless, applicable to all states of matter in different forms without special requirements for sample preparation, independent on chemicals, usable as in situ analysis, usable for measuring through transparent glass or polymeric covering layers. Since the Raman scattering is a weak effect, some adverse effects, such as luminescence, can influence the quality of spectral response and mask spectral information.

Raman spectra were measured using Renishaw InVia Basis Raman microscope with NIR diode laser (785 nm) with maximum output power $300 \mathrm{~mW}$. Leica DM 2500 confocal microscope with the resolution $2 \mu \mathrm{m}$ was coupled to the spectrometer. All measurements were collected with $4 \mathrm{~s}$ exposure time and 15 accumulations. The samples were firstly scanned in the range from 100 to $3200 \mathrm{~cm}^{-1}$. A spectral range for the evaluation was reduced to $700-1800 \mathrm{~cm}^{-1}$.

\subsection{Multivariate analysis techniques}

Raman spectroscopic data are multivariate, since they reflect the composition of material and its structure. Raman peaks are assigned to vibrations of chemical bonds in the analysed materials. For the evaluation it is appropriate to use multivariate mathematics. Methods that enable multivariate analysis such as principal component analysis and cluster analysis were used for data mining from the large spectral data sets to find the patterns within the data.

\section{Results}

Raman spectra of the textile fibres were measured on Raman microscope. A part of the obtained spectra is shown in Fig. 1a. The only spectrum that was excluded from the evaluation is the spectrum of viscose, because of its high luminescence. The linear baseline correction and unit normalization was applied on the spectra for further multivariate statistical evaluation. The data were analysed by principal component analysis, the output is displayed in Fig. 1b. The scores plot clearly shows the formation of three groups. Closer look at each of the groups obviously indicate the


Fig. 1. a) Raman spectra of cotton, ramie, flax, lyocell, nylon, polyamide, merino wool, silk (top to bottom). b) Principal component analysis of the Raman data of fibres 


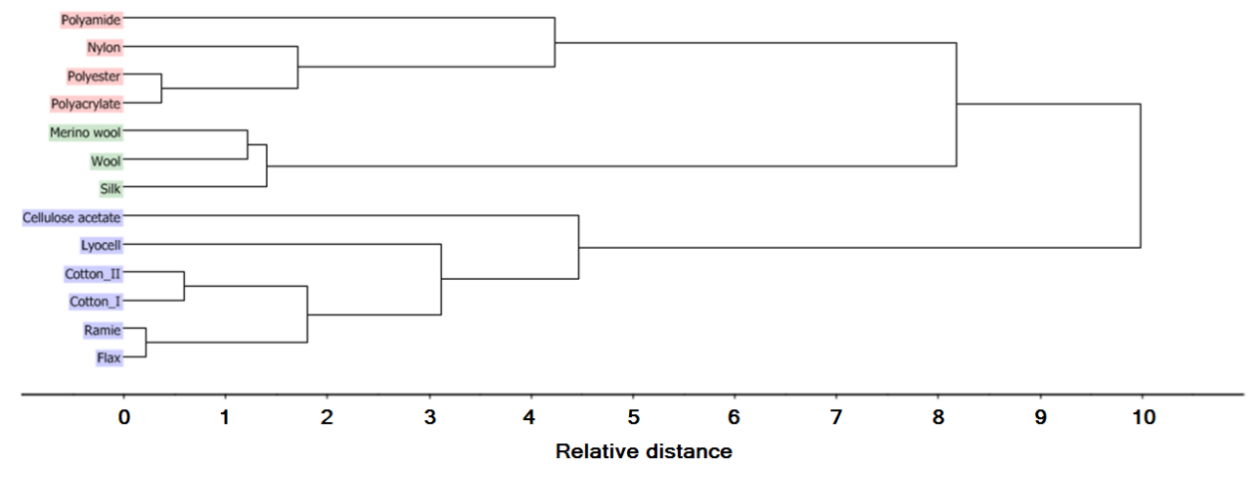

Fig. 2. Cluster analysis of the of the Raman data of fibres

differences associated with the source of the fibres - natural vegetable (based on cellulose), natural animal (based on protein) and man-made non-cellulosic polymers.

The hierarchical complete linkage based on correlation distance measure was used for the cluster analysis. There is the dendrogram in Fig. 2 which also shows the differentiation to three main sub-clusters according to nature of the fibre origin. An exception in the grouping could be lyocell and cellulose acetate. Although man-made fibres, these two samples are linked to the natural vegetable samples. The explanation arises from their cellulose source.

The database of Raman spectra of fibres was created on the bases of acquired data. The database was successfully tested on other samples of fibres with the known origin and composition. The database serves as a tool for identification of unknown samples. Samples can be due to advantages of Raman spectroscopy and coupled microscopy in the form of solitary pieces of fibre in dimensions in the order of micrometres. The database is helpful for identifying fibres as forensic traces.

\section{Conclusion}

Analytical method Raman spectroscopy was chosen for the examination of textile fibres. Data reflecting structural differences of thirteen textile fibres were acquired by this modern method. The evaluation of the datasets was performed using multivariate statistical methods principal component analysis and cluster analysis. Raman spectra show visible differentiation. The statistical results indicate agreement with the nature of the fibre origin. The database of Raman spectra was created for further identification of fibres using Spectral ID software. Forensic demonstration was done, however, the results will be published separately. Up to now only natural/white fibres were studied. The future perspective is to focus on enlarging the number of measured materials, on dyed fibres and another forensic application.

\section{Acknowledgments}

This work was supported by the Ministry of Education, Youth and Sports of the Czech Republic within the National Sustainability Programme project No. LO1303 (MSMT-7778/2014) and also by the European Regional Development Fund under the project CEBIA-Tech No. CZ.1.05/2.1.00/03.0089.

\section{References}

[1] Kvavadze, E., Bar-Yosef, O., et.al. (2009). 30,000-year-old wild flax fibers. Science, Vol. 325, pp. 1359-1359.

[2] Simoncic, B., \& Tomsic, B. (2010). Structures of novel antimicrobial agents for textiles-a review. Textile Research Journal, Vol. 80, No. 16, 2010, pp. 1721-1737.

[3] Glogar, M. I., Parac-Osterman, Đ., Grundler, D., \& Rolich, T. (2011). The rule-based approach in studying the influence of a different textile surfaces on colour similarity. In 22nd International DAAAM Symposium" Intelligent Manufacturing \& Automation", pp 1207-1208.

[4] Muehlethaler, C., Leona, M., \& Lombardi, J. R. (2015). Review of surface enhanced Raman scattering applications in forensic science. Analytical chemistry, Vol. 88, No.1, 2015, pp.152-169.

[5] Kavkler, K., \& Demšar, A. (2011). Examination of cellulose textile fibres in historical objects by micro-Raman spectroscopy. Spectrochim Acta A Mol Biomol Spectrosc, Vol. 78, No. 2, 2011, pp. 740-746.

[6] Ivanov, R., Hussainova, I., Aghayan, M., \& Petrov, M. (2014). Graphene coated alumina nanofibers as zirconia reinforcement. In 9th Int. DAAAM Balt. Conf, Vol. 348, 2014, pp. 348 - 353.

[7] Neumann, P., Houser, J., Pospíšilík, M., Skočík, P., \& Adámek, M. (2015) Some methods for electronic component authenticity assessment. In Annals of DAAAM and Proceedings of the International DAAAM Symposium, pp.111.

[8] Chalmers, J. M., Howell G. E. \& M. D. Hargreaves, Infrared and Raman spectroscopy in forensic science. 1st pub. Chichester, West Sussex, UK: Wiley, 2012 